\title{
FACTORES MATERNOS ASOCIADOS A MACROSOMIA FETAL EN UN HOSPITAL PÚBLICO DE LIMA- PERÚ, ENERO A OCTUBRE DEL 2018
}

\author{
MATERNAL FACTORS ASSOCIATED WITH FETAL MACROSOMIA IN A PUBLIC HOSPITAL OF LIMA- PERU, \\ JANUARY TO OCTOBER 2018 \\ Dania Bazalar-Salas ${ }^{1, a}$, María Loo-Valverde ${ }^{2, b}$
}

\begin{abstract}
RESUMEN
Objetivo: Determinar los factores maternos asociados a macrosomia fetal en un Hospital Público de Lima - Perú, de enero a octubre del 2018. Métodos: Estudio analítico, observacional, retrospectivo, de tipo casos y controles. Se utilizó una ficha de recolección de datos y para el análisis estadístico se utilizó el programa SPSS v25.0. Resultados: De los 401 recién nacidos, el 27,2\% de las madres estudiadas tuvo una ganancia de peso inadecuado ( $O R=6.96, I C 95 \% 4.22-11.48, p=0.00)$, en cuanto a la categoría de peso pregestacional, y la paridad fue de 73 (73.7\%), Asimismo, la edad materna fue menor de 35 años en 278 $(92.1 \%)$, siendo estadísticamente significativa $(\mathrm{p}=0.004)$. Conclusión: Se concluye que, existe relación de los factores maternos como lo fueron ganancia de peso inadecuado durante la gestación, peso pre gestacional materno, paridad, edad materna asociado con la macrosomia fetal.
\end{abstract}

Palabras clave: Macrosomia Fetal; Factores de riesgo; Paridad; Edad gestacional. (fuente: DeCS BIREME)

\begin{abstract}
Objective: To determine the maternal factors associated to fetal macrosomia in a Public Hospital of Lima - Peru, from January to October of 2018. Methods: Analytical, observational, retrospective study, of type cases and controls. A data collection form was used and for the statistical analysis the SPSS V25.0 program was used. Results: Of the 401 newborns, it was found that the weight gain during pregnancy $27.2 \%$ the mothers studied had an inadequate weight gain ( $\mathrm{OR}=6.96,95 \% \mathrm{Cl} 4.22-11.48, \mathrm{p}=0.00)$, regarding the prepregnancy weight category, and parity was $73(73.7 \%)$. Also, maternal age was less than 35 years in 278 (92.1\%), being statistically significant $(p=0.004)$. Conclusion: It is concluded that there is a relationship of maternal factors such as inadequate weight gain during pregnancy, maternal pre-gestational weight, parity, maternal age associated with fetal macrosomia.
\end{abstract}

Key words: Fetal Macrosomia; Risk factors; Parity; Gestational age. (source: MeSH NLM)

\section{INTRODUCCIÓN}

La Macrosomía' se define como un desarrollo excesivo de acuerdo al peso del Recién Nacido superior a 4500 gramos y para otros es útil el uso del percentil 90, sin embargo, en estos últimos años la frecuencia de casos de macrosomia se incrementó en países desarrollados económicamente, $5.4 \%$ con un peso superior a 4500 gramos y de $10 \%$ a $13 \%$ con un peso superior o igual a 4000 gramos $^{2}$.
En los últimos años, la incidencia de macrosomia ha aumentado considerablemente, reportándose tasas que oscilan entre el $10 \%$ y el $13 \%$ cuando se utiliza como valor neto un peso de nacimiento superior a 4,000 gramos ${ }^{3}$. En la actualidad, además de peso al nacimiento (PN) y de la edad gestacional (EG), se propone el índice ponderal (IP) como un parámetro que definiría dos subtipos de macrosómicos.

\footnotetext{
${ }^{1}$ Hospital Sergio Bernales, Lima-Perú.

${ }^{2}$ Cardióloga pediatra del Hospital Nacional Edgardo Rebagliati Martins, Lima-Perú.

a Médico Interna.

${ }^{\text {b}}$ Cardióloga pediatra.
}

Citar como: Dania Bazalar-Salas, María Loo-Valverde. Factores maternos asociados a macrosomia fetal en un hospital público de Lima- Perú, enero a octubre del 2018. [Artículo Original].2019;19(2):00-00. (Abril 2019). DOI 10.25176/RFMH.v19.n2.2066 
En la actualidad, la prevalencia de macrosomia fetal varía de 4.1 a $13.4 \%$ en diferentes grupos étnicos ${ }^{4,5}$. Estos y otros estudios refieren que los principales factores asociados con el incremento de la macrosomia son la diabetes materna, el embarazo prolongado, la ganancia de peso aumentado durante el embarazo y el IMC incrementado, entre otros ${ }^{6,7}$.

La Organización Mundial de la Salud (OMS), en un estudio realizado entre el 2006 y el 2012, muestra cifras de $7,6 \%$ a nivel de Sudamérica, de recién nacidos con sobrepeso mientras que, en el Perú las cifras fueron de $6,9 \% 9,10$.

En el Perú, en estudios realizados por el Dr. Rendón ${ }^{11}$ muestran cifras de macrosomia fetal en hospitales del Ministerio de Salud del Perú de 11,37\%, donde se destaca la variación de porcentajes según las regiones del interior de nuestro país, mostrándose una mayor prevalencia en la región Costa con un $14,34 \%$, la sierra con $7,12 \%$ y la selva con $9,81 \%$, lo cual refuerza la asociación de la misma, con las condiciones del ámbito social y económico de la población ${ }^{11}$.

El objetivo principal del presente estudio fue determinar los factores maternos asociados a macrosomia fetal en el servicio de ginecología y obstetricia de un Hospital Público de Lima - Perú de enero a octubre del 2018.

\section{MÉTODOS}

Se realizó un estudio de tipo analítico, observacional, retrospectivo, de tipo casos y controles, de enfoque cuantitativo y de estadística inferencial, donde se recopiló información proveniente de los recién nacidos del servicio de ginecología y obstetricia del Hospital Sergio Bernales durante el período de enero a octubre del 2018.

La población de estudio estuvo conformada por 3150 recién nacidos, de acuerdo a los criterios de selección, se recolectó la información de 401 recién nacidos, con 99 casos y 302 controles, debido a que se excluyeron recién nacidos macrosómico transferido de otro centro hospitalario, recién nacidos de madres con un peso pregestacional dentro de la categoría bajo peso y madres con una ganancia de peso por debajo de lo esperado para la categoría de peso pregestacional.

La información obtenida de las historias clínicas y libros de registro de recién nacidos se recolecto en una ficha de datos, posteriormente se colocó en una base de datos y se utilizó un software para el procesamiento de datos, el programa SPSS Statistics versión 25 .
Para el análisis estadístico de los datos, se calculó del Odds Ratio con sus propios intervalos de confianza $95 \%$ y una significancia $\mathrm{p}(<0.05)$ mediante la prueba de Chi.-cuadrado.

\section{RESULTADOS}

De 3150 recién nacidos la prevalencia fue de 5.65\% que tuvieron macrosomia fetal, tanto por parto vaginal, como por cesárea, según los criterios de selección la muestra fue de 401 recién nacidos: 99 casos y 302 controles.

En cuanto a los factores maternos asociados a macrosomia fetal, se encontró que la ganancia de peso durante la gestación; de nuestra muestra estudiada $251(83.1 \%)$ fue adecuada, en cuanto a la categoría de peso pregestacional, siendo predominante sobrepeso u obesidad, y la paridad, encontrándose prevalencia en pacientes multíparas fue de 73 (73.7\%), respectivamente. Asimismo, la edad materna fue menor de 35 años en 278 (92.1\%). Finalmente, 291 (96.4\%) de las pacientes no tuvieron antecedentes de macrosomia fetal. Tabla 1.

\section{DISCUSIÓN}

La macrosomia se establece como diagnóstico no solo a nivel nacional, sino también internacional, según Cunha et $\mathrm{al}^{12}$, en nuestro país la incidencia fue 5.3\% en el año 2012, otros estudios realizados en hospitales del interior del país como el de Quiroz F. Rolando ${ }^{13}$ en el Hospital María Auxiliadora en el año 2016, demostró una incidencia de $9.83 \%$, mientras que en otro estudio realizado por Paico L. Daniel ${ }^{14}$ en el mismo hospital en el año 2017 encontró una incidencia de 9.1\%; en el presente estudio se encontró una incidencia de $6 \%$ de macrosomia fetal en el Hospital Sergio Bernales, lo cual indicaría que la prevalencia de macrosomia del mencionado hospital se encuentra cercana a los valores nacionales y por debajo de la prevalencia de otros hospitales.

De acuerdo al estudio realizado por García, Ángel R. ${ }^{15}$, en el servicio de obstetricia del hospital Félix Mayorca Soto de Tarma en el 2014, observaron que los productos Macrosómicos en su mayoría eran de madres con obesidad, sin embargo, los resultados no eran estadísticamente significativos, un estudio realizado por Herrera Risco16 en el Centro Materno Infantil César López Silva del distrito de Villa El Salvador año 2011, donde el índice de masa corporal (IMC) promedio fue de $23.6 \pm 3.1 \mathrm{Kg} / \mathrm{m}^{2}$, encontró una correlación baja pero significativa entre la ganancia 
Tabla 1. Características generales de los factores maternos asociados a macrosomía fetal.

\begin{tabular}{|c|c|c|c|c|c|c|c|}
\hline & & CASOS & CONTROL & OR & \multicolumn{2}{|c|}{ IC $95 \%$} & $\mathbf{P}$ \\
\hline \multirow{2}{*}{$\begin{array}{l}\text { Ganancia de peso } \\
\text { durante la gestación }\end{array}$} & No adecuado & $58(58,6 \%)$ & $51(16,9 \%)$ & \multirow{2}{*}{6,96} & \multirow{2}{*}{4,22} & \multirow{2}{*}{11,48} & \multirow{2}{*}{0,00} \\
\hline & Adecuado & $41(41,4 \%)$ & $251(83,1 \%)$ & & & & \\
\hline \multirow{2}{*}{$\begin{array}{l}\text { Categoría de peso } \\
\text { pregestacional }\end{array}$} & Sobrepeso u obesidad & $73(73,7 \%)$ & $62(20,5 \%)$ & \multirow{2}{*}{10,86} & \multirow{2}{*}{6,41} & \multirow{2}{*}{18,42} & \multirow{2}{*}{0,00} \\
\hline & Normal & $26(26,3 \%)$ & $240(79,5 \%)$ & & & & \\
\hline \multirow{3}{*}{ Paridad } & Multípara o gran multípara & $73(73,7 \%)$ & $160(53,0 \%)$ & \multirow{3}{*}{2,49} & \multirow{3}{*}{1,5} & \multirow{3}{*}{4,11} & \multirow{3}{*}{0,00} \\
\hline & Drimín & $26(2620)$ & $112(1700$ & & & & \\
\hline & & & & & & & \\
\hline \multirow{2}{*}{ Edad materna } & Mayores de 35 años & $18(18,2 \%)$ & $24(7,9 \%)$ & \multirow[b]{2}{*}{2,57} & \multirow[b]{2}{*}{1,33} & \multirow[b]{2}{*}{4,97} & \multirow[b]{2}{*}{0,004} \\
\hline & Menores o igual a 35 años & $81(81,8 \%)$ & $278(92,1 \%)$ & & & & \\
\hline \multirow{2}{*}{$\begin{array}{l}\text { Antecedente de } \\
\text { macrosomía fetal }\end{array}$} & $\mathrm{Si}$ & $69(69,7 \%)$ & $291(96,4 \%)$ & \multirow{2}{*}{11,5} & \multirow{2}{*}{5,49} & \multirow{2}{*}{24,08} & \multirow{2}{*}{0,00} \\
\hline & No & $30(30,3 \%)$ & $11(3,6 \%)$ & & & & \\
\hline \multicolumn{2}{|l|}{ TOTAL } & 99 & 302 & & & & \\
\hline
\end{tabular}

Fuente: Instituto de Investigación de Ciencias Biomédicas (INICIB).

En el análisis multivariado, se encontró que las variables de los factores maternos se mantuvo asociado con macrosomía fetal, siendo estadísticamente significativa $(p=0.00, p=004)$, tabla 2 .

Tabla 2. Análisis multivariado de los factores maternos asociados a macrosomía fetal.

\begin{tabular}{lccc}
\multicolumn{1}{c}{ VARIABLES } & OR & IC 95\% & P \\
\hline Ganancia de peso durante la gestación & 5,49 & $3,07-9.81$ & 0,00 \\
Categoría de peso pregestacional & 9,4 & $5,28-16,72$ & 0,00 \\
Paridad & 2,28 & $1,24-4,1$ & 0,00 \\
Edad materna & 2,33 & $1,009-5,38$ & 0,04 \\
\hline
\end{tabular}

de peso de la madre y el peso del recién nacido, de igual manera en el estudio realizado en el Hospital Sergio Bernales año 2016 por Sotomayor B. Joanna ${ }^{17}$ se describe una relación estadísticamente significativa entre la ganancia del peso materno y el peso del recién nacido, sin embargo en el mencionado estudio se tomó una población muestral pequeña.

En el estudio realizado por Jiménez Puñales Sandi' ${ }^{18}$ y colaboradores predomina la obesidad materna: $85(94.3 \%)(p=0.003)$, como factor de riesgo para un producto macrosómico, corroborando lo hallado en nuestro estudio, siendo este un factor de riesgo controlable que con una correcta orientación materna disminuiría la tasa de macrosomia fetal, evitando así exponer al recién nacido a las complicaciones a que este problema conlleva. Asimismo, un factor de riesgo para la macrosomia fetal, también es la paridad, ya que estudios han demostrado que a mayor número de gestaciones la probabilidad de productos Macrosómicos se incrementa ${ }^{19}$ hasta en un $70 \%$, en el presente estudio un $73.7 \%$ de las madres de productos Macrosómicos son multíparas o gran multíparas, respaldando así los estudios previos, ya que se halló significancia estadística con respecto a esta variable.

La edad materna es otro factor de riesgo para macrosomia fetal, algunos autores atribuyen esto 
al metabolismo materno relacionándolo con el crecimiento intrauterino del feto ${ }^{16}$; en el estudio realizado por Alejandro Estrada y colaboradores ${ }^{15}$ se demostró que el tener 35 años o más es un factor de riesgo para macrosomia fetal (OR $=1.1$, IC95\%: 1.11.1), sin embargo en nuestro estudio se encontró que las pacientes que tenían edades menores o iguales a 35 años tuvieron un producto macrosómico.

Con respecto al antecedente de macrosomia fetal, se menciona que el riesgo de macrosomia fetal se incrementa en cada gestación, de esa manera y teniendo como límite el quinto embarazo, se refiere que el peso promedio al nacimiento en cada gestación se incrementa progresivamente aproximadamente unas 4 onzas lo que equivale a $113 \mathrm{~g}^{19}$, lo cual se correlaciona con los resultados de este estudio, en donde en el análisis bivariado se encontró correlación entre antecedente de macrosomia fetal y un nuevo producto macrosómico, siendo estadísticamente significativo $(p=0.00)$.

\section{CONCLUSIÓN}

Se concluye que, las variables de los factores maternos como: ganancia de peso inadecuado durante la gestación, peso pregestacional materno, paridad y edad materna están asociadas con la macrosomia fetal.

Contribuciones de autoría: Los autores participaron en la génesis de la idea, diseño de proyecto, recolección e interpretación de datos, análisis de resultados, preparación del manuscrito.

Financiamiento: Autofinanciado.

Conflicto de interés: Los autores declaran no tener conflicto de interés en la publicación de este artículo.

Recibido: 12 de noviembre 2018

Aprobado: 17 de enero de 2019

Correspondencia: Dania Lysbeth Bazalar Salas

Dirección: Jr. Manuel Puerto Carrero 851. Zona D. San Juan de Miraflores, Lima-Perú

Teléfono: +51943459843

Correo:danialisbeth@hotmail.com

\section{REFERENCIAS BIBLIOGRÁFICAS}

1. American College of Obstetrics and Gynecologist. Fetal Macrosomia Practice Bulletin. EEUU: Ginecología Medica. 2000.

2. Razzo, L. Incidencia y factores de riesgo de macrosomia fetal en el Hospital San José del Callao: Enero - Diciembre 2006. [Tesis para Médico Cirujano] Lima: Universidad San Marcos; 2014.

3. Allahyar Jazayeri, MD, PhD. Macrosomia. Medicine 2007. Disponible: http://www.emedicine.com/med/topic3279.htm

4. Sinclair BA, Rowan JA, Hainsworth OT. Macrosomic infants are not all equal. Aust N Z J Obstet Gynaecol 2007; 47: 101-105.

5. Li G, et al. Prevalence of macrosomia and its risk factors in china: a multicentre survey based on birth data involving 101,723 singleton term infants. https://doi.org/10.1111/ ppe.12133.

6. Cunha $A$, et al. Prevalencia y factores asociados a macrosomia en Perú, 2013. Disponible en: https://doi.org/10.17843/ rpmesp.2017.341.2765

7. Soltani $\mathrm{H}$, et al. Pre-pregnancy body mass index and gestational weight gain and their effects on pregnancy and birth outcomes: a cohort study in West Sumatra, Indonesia. Disponible en: https://doi.org/10.1186/ s12905-017-0455-2

8. Crane JM, Blanco J, Murphy P, Burrage L, Hutchens D. The effect of gestational weight gain by body mass index on maternal and neonata outcomes. J Obstet Gynaecol Can 2009; 31(1): 28-35

9. Sotomayor B. Joanna. Relación entre la ganancia de peso materno y el peso de recién nacido en gestantes a término atendidas en el Hospital Nacional Sergio E. Bernales - Comas. Perú: Universidad San Martin de Porres; 2016,43 p.

10. Chávez A. Katherin. Factores maternos asociados a macrosomia en recién nacidos de puérperas atendidas en el Hospital Nacional Sergio E. Bernales, mayo - octubre 2014.

11. Rendón MT, Apaza DH. Macrosomia fetal en el Perú: prevalencia factores de riesgo y resultados perinatales. Ciencia y Desarrollo, 17 febrero de 2017;10(10).
12. Mardones Francisco, et al. Influencia combinada del índice de masa corporal pregestacional y de la ganancia de peso en el embarazo sobre el crecimiento fetal. Rev. méd. Chile. 2011 jun; 139(6): 710-716.

13. García Orosco, Ángel R., Relación entre la ganancia de peso materno y macrosomía fetal, Lima - Perú: Universidad Mayor de San Marcos, 2014. $35 \mathrm{p}$.

14. Recién nacido de peso elevado A. Aguirre Unceta-Barrenechea, A Aguirre Conde, A. Pérez Legórburu, I. Echániz Urcelay Unidad Neonatal. Servicio de Pediatría. Hospital de Basurto. Bilbao.

15. Alejandro Estrada et al. Factores maternos relacionados con el peso al nacer de recién nacidos a término, Colombia, 2002-2011. Cad. Saúde Pública 32 (11) 08 Dic 2016.

16. Quiroz Flores, Rolando S. Factores de riesgo para macrosomia fetal en el Hospital María Auxiliadora: enero - diciembre 2016. Lima -Perú: Universidad Ricardo Palma; 2016. 67 p

17. Toirac Lamarque Abelardo Salvador, et al. Macrosomia fetal en madres no diabéticas. Caracterización mínima. MEDISAN [Internet]. 2013 Oct [citado 2019 Feb 17] ; 17(10): 6052-6062.

18. Jiménez Puñales Sandi y colaboradores. Factores de riesgo maternos y fetales en recién nacidos con macrosomia. Medicentro Electrónica. 2015 Sep; 19(3): 142-148.

19. Paico L. Daniel, Factores predisponentes de macrosomía en el Hospital de Vitarte durante el periodo de julio - diciembre 2016. Perú: Universidad Ricardo Palma; 2017, 56 p.

20. Susanibar Peña C. Factores asociados a morbimortalidad en recién nacidos macrosómicos atendidos en el hospital vitarte en el periodo enero a diciembre del 2014 .2016. Disponible en: http://alicia.concytec. gob.pe/vufind/Record/URPU_c32317f5f702a783 e048330725a8d896.

21. Minjarez-Corral M, et al. Ganancia de peso gestacional como factor de riesgo para desarrollar complicaciones obstétricas. Perinatal Reprod Humana. Septiembre de 2014;28(3):159-66. 